\title{
INFECCIÓN POR ORBIVIRUS EN HUANGANAS (Tayassu pecari) DE MADRE DE DIOS
}

\author{
Orbivirus Infection in White-Lipped Peccaries (TAyassu Pecari) from Madre \\ de Dios Region, Peru
}

Hermelinda Rivera G. ${ }^{1,3}$, Luis Cárdenas P. ${ }^{1}$, Mercy Ramírez V. ${ }^{1}$, Alberto Manchego S. ${ }^{1}$, Juan More B. ${ }^{1}$, Alfonso Zúñiga H. ${ }^{2}$, Mónica Romero S. ${ }^{2}$

\section{Resumen}

El Perú posee el 13\% de los bosques tropicales amazónicos con más de 460 especies de mamíferos, incluyendo el pecarí labiado o huangana (Tayassu pecari). Esta especie es de gran importancia ecológica y comercial, así como una importante fuente de proteína para la población amazónica; sin embargo, las informaciones sobre su estado sanitario son escasas. El objetivo del estudio fue determinar anticuerpos contra el virus de lengua azul (VLA) y otros Orbivirus en huanganas de vida libre de apariencia normal de tres localidades del departamento de Madre de Dios. Se evaluaron 106 muestras de suero para determinar los anticuerpos contra el VLA mediante un kit de ELISA de competición y contra otros Orbivirus mediante la prueba de inmunodifusión en gel de agar. El 7.5\% (8/106) presentaron anticuerpos específicos contra el VLA y el 29.2\% (31/106) contra VLA/otro Orbivirus. Anticuerpos contra el VLA y VLA/otro Orbivirus se hallaron en las tres localidades.

Palabras clave: huangana, Tayassu pecari, virus de lengua azul, orbivirus, anticuerpos, inmunodifusión, ELISA de competición, Amazonía peruana

\section{Abstract}

Peru has $13 \%$ of the Amazonian tropical forest which contains over 460 species of mammals, including the white lipped peccary or huangana (Tayassu peccari). This species is of great ecological and commercial importance and a major source of protein for the local inhabitants; however, information about the sanitary situation of this species is scarce. The aim of this study was to determine antibodies against bluetongue virus

\footnotetext{
${ }^{1}$ Laboratorio de Microbiología y Parasitología Veterinaria, Facultad de Medicina Veterinaria, Universidad Nacional Mayor de San Marcos, Lima

2 Proyecto ÁREAS-Amazonía de la World Wildlife Fund (WWF-Peru)

${ }^{3}$ E-mail: hriverag2005@yahoo.es
} 
(BTV) and other Orbivirus in free-living and healthy white lipped peccaries from the Madre de Dios region, Peru. One hundred and six serum samples were evaluated to determine antibodies against BTV by competitive ELISA and other Orbivirus by immunodiffusion agar gel test. Results showed that $7.5 \%$ (8/106) of the samples had antibodies against BTV and 29.2\% (31/106) against BTV/other Orbivirus. Antibodies against BTV and other Orbivirus were detected in all three localities.

Key words: white-lipped peccary, Tayassu peccari, bluetongue virus, Orbivirus, antibodies, immunodiffusion, competitive ELISA, Peruvian Amazon

\section{INTRODUCCIÓN}

La conservación de la fauna silvestre requiere de adecuadas políticas de gobierno y estudios que contribuyan al conocimiento de la biología, estado sanitario, importancia ecológica, etc. El Perú es uno de los cinco países con mayor diversidad biológica, siendo el segundo en América Latina y el cuarto a nivel mundial en riqueza de bosques tropicales, pues posee el $13 \%$ de los bosques tropicales amazónicos (Congreso de la República. Comercio Exterior y Turismo, 2004). En términos de fauna, posee más de 460 especies de mamíferos, incluyendo el pecarí labiado o huangana (Tayassu pecari).

En el país se han llevado a cabo algunos estudios sobre la biología, conducta poblacional y perfiles bioquímicos de la huangana (Painter, 1996; Herrera et al., 2008; Romero et al., 2008), pero a pesar de su importancia socioeconómica para el poblador amazónico y ser parte de la biodiversidad, son escasas las informaciones sobre su aspecto sanitario y de su rol como reservorio de agentes infecciosos para animales domésticos y el hombre. Recientemente, Romero (2010) evaluó algunas enfermedades bacterianas, parasitarias y virales que afectan al porcino doméstico en muestras de huanganas pero no incluyó al virus de lengua azul. Este es un Arbovirus endémico en zonas tropicales y subtropicales y actualmente en áreas templadas, debido posiblemente a la incursión de insectos vectores en otras latitudes (García et al., 2009).
El virus de Lengua Azul (VLA) es miembro del género Orbivirus de la familia Reoviridae. En el género Orbivirus hay 19 serogrupos, siendo uno de ellos el serogrupo del VLA, que a su vez posee 26 serotipos (MacLachlan, 2011; Savini et al., 2011) que comparten antígenos detectables por pruebas de inmunodifusión, fijación de complemento e inmunofluorescencia. Esto implica reacción cruzada con otros serogrupos como el virus de Enfermedad Hemorrágica Epizoótica (EHDV, por sus siglas en inglés) y otros virus aunque en menor grado (DellaPorta et al., 1985; Mertens et al., 2004; Kedmi et al., 2011).

El VLA y EHDV se trasmiten a rumiantes domésticos y silvestres y otros mamíferos a través de insectos del género Culicoides. Estos virus comparten signos clínicos y ecosistemas originando un problema para el diagnóstico clínico y de laboratorio si se utilizan técnicas convencionales como la inmunodifusión (Stallknecht et al., 1996; Savini et al., 2011).

La prueba de inmunodifusión en gel de agar (IDGA) fue utilizada ampliamente para el diagnóstico de VLA como una prueba estándar en el comercio internacional (Pearson et al., 1985), e incluso se sigue empleando con ese propósito por ser una prueba simple y económica. Della-Porta et al. (1985) reportaron, sin embargo, que la IDGA presentaba reacción cruzada entre el VLA y EHDV y, en menor grado, con los virus Eubenangœ y Paliam, también miem- 
bros del género orbivirus. Esto se debe a que los anticuerpos contra el VLA detectan antígenos compartidos presentes en la nucleocápside viral (VP3 y VP7), sugiriendo que estas reacciones cruzadas pueden ser indicativo de una ruta evolucionaria común entre los virus mencionados (Gorman, 1986; Ristow et al., 1988).

Actualmente, se dispone de técnicas más sensibles y específicas para los estudios serológicos de VLA como la ELISA de competición que detecta anticuerpos específicos contra el VLA y las técnicas moleculares para detección de genotipos (Clavijo et al., 2010; Madani et al., 2011). A diferencia, aún no existen pruebas serológicas comerciales para el diagnóstico específico de EHDV. La Organización Mundial de Sanidad Animal (OIE, 2008) incluye al VLA y EHDV en el Manual de Técnicas Diagnósticas y Vacunas sin describir una técnica diagnóstica específica.

En el Perú, la infección por VLA ha sido reportada serológicamente en ovejas (Rosadio et al., 1984), bovinos y otras especies como el sajino (Tayassu tajacu) en zoocriaderos en el trópico y en venados en el norte del país, mediante la prueba de IDGA (Rivera, datos no publicados), pero no existen informaciones serológicas ni clínicas sobre VLA y EHDV. El objetivo del presente estudio fue determinar anticuerpos contra el VLA y otros Orbivirus en huanganas de vida libre en la zona de Madre de Dios, en el contexto de vigilancia de agentes virales transmitidos por insectos.

\section{Materiales y MéTodos}

Se recolectaron muestras de sangre de huanganas de apariencia normal, capturadas cerca o dentro de tres áreas protegidas del departamento de Madre de Dios: Boca de Manu, Concesión para la Conservación Los Amigos y Reserva Nacional Tambopata/Parque Nacional Bahuaja Sonene, en el periodo
2008-2009. El grupo de investigadores contó con los permisos de la Dirección General Forestal y de Fauna Silvestre (DGFFS) del Ministerio de Agricultura y de las jefaturas de las respectivas áreas naturales protegidas.

El área de estudio se encuentra a una altitud de 200-350 msnm, presenta clima tropical húmedo, con época seca entre junio a septiembre y con los meses de mayor intensidad de lluvias entre diciembre y febrero. La precipitación anual promedio es de 2500 a 3500 mm y la temperatura ambiental de $24^{\circ} \mathrm{C}$, con rangos de 10 a $38^{\circ} \mathrm{C}$. Se encuentra ubicada entre los $12-13^{\circ}$ latitud sur y $69-70^{\circ}$ longitud oeste (Tobler et al., 2009).

Las muestras obtenidas en la Concesión para la Conservación del Río Los Amigos y en la Reserva Nacional Tambopata/ Parque Nacional Bahuaja Sonene fueron colectadas durante la captura de animales para la colocación de radiotransmisores por el proyecto ÁREAS-Amazonía de la World Wildlife Fund (WWF-Perú), usando redes de caída e inmovilizando a los animales con midazolam $(0.2-0.3 \mathrm{mg} / \mathrm{kg})$ y medetomidina $(0.04-0.06 \mathrm{mg} / \mathrm{kg})$ por cerca de 60 minutos, y aplicándoles posteriormente los antagonistas específicos atipamezol $(0.2-0.3 \mathrm{mg} / \mathrm{kg}) \mathrm{y}$ flumazenil $(0.01 \mathrm{mg} / \mathrm{kg}$ ) (Zúñiga et al, datos no publicados). Las muestras de Boca Manu provinieron de animales cazados para consumo por miembros de la comunidad nativa, donde la toma de sangre se hizo inmediatamente de caer abatido el animal (Romero, 2010).

Se colectaron 106 muestras de sangre de la vena cefálica o de la safena utilizando agujas y tubos al vacío. El suero fue obtenido por centrifugación a $800 \mathrm{G}$ por 10 minutos en el campamento del proyecto y envasados en viales y conservados a $-20^{\circ} \mathrm{C}$. Las muestras fueron enviadas a la Facultad de Medicina Veterinaria de la Universidad Nacional Mayor de San Marcos, Lima.

Para la detección de anticuerpos contra el VLA se utilizó un kit de ELISA de com- 
petición (VMRD, EEUU), que posee una sensibilidad de $100 \%$ y especificidad de $99 \%$. El kit contiene un antígeno común a los 25 serotipos de VLA y un anticuerpo monoclonal contra la proteína VP7 que tiene los epitopos que determinan la especificidad del serogrupo del VLA. En el desarrollo de la prueba se siguieron las instrucciones del fabricante.

La detección de anticuerpos contra el VLA y otros Orbivirus se hizo con un kit comercial de IDGA (VMRD, EEUU), con sensibilidad y especificidad de 100 y $99 \%$, respectivamente, según indica el fabricante. El kit contiene un antígeno común al serogrupo del VLA, con reacción cruzada con otros miembros del género Orbivirus, incluyendo el EHDV (Chandel et al., 2003).

\section{Resultados y Discusión}

El 7.5\% (8/106) de las muestras tuvieron anticuerpos específicos contra el VLA y el 29.2\% (31/106) tuvieron anticuerpos contra el serogrupo VLA pero que presenta reacción cruzada con otros orbivirus (Cuadro 1). Huanganas de las tres áreas mencionadas presentaron anticuerpos específicos y no específicos contra el VLA.

Estudios epidemiológicos indican que el VLA existe en una extensa zona en el mundo entre los $40^{\circ}$ latitud norte y $35^{\circ}$ latitud sur, con ecosistemas tropicales, subtropicales y templados (MacLachlan, 2004; Tabachnick, 2004), y la mayoría de los países de América Latina, incluyendo el Perú, están dentro de esos límites (Lager et al., 2004). Estudios serológicos efectuados en bovinos, caprinos, ovinos y búfalos en Argentina, Brasil, Colombia y Venezuela indican variadas prevalencias que van $0.7 \%$ hasta más del $80 \%$ (Lager, 2004). En el Perú, Rosadio et al. (1984) reportaron una seroprevalencia de 88,41 y $56 \%$ del VLA en ovejas del norte, centro y sur de la sierra del país, respectivamente, y Rivera et al. (1987) reportaron $21 \%$ de animales serorreactores en alpacas del sur del país.
No obstante, la mayoría de estos estudios fueron realizados con la prueba de IDGA, por lo que es muy probable que los anticuerpos detectados y reportados contra el VLA fueran también contra otros Orbivirus relacionados como el EHDV. Cabe destacar las diferencias en seroprevalencia detectada en el presente estudio con el empleo del kit específico (7.5\%) y con el kit de IDGA (29.2\%).

Estudios serológicos realizados en animales de vida silvestre en América Latina son escasos. Gerber et al. (2012) utilizando la prueba de IDGA reportaron 39\% (19/49) de sajinos (Tayassu tajacu) positivos a Orbivirus en Brasil; así mismo en sajinos de un zoocriaderos en Iquitos y en otro en Pucallpa (Ucayali) se encontró 19\% (4/21) y $56 \%(39 / 68)$ de serorreactores, respectivamente, mediante la prueba de IDGA ( $\mathrm{H}$. Rivera, trabajo no publicado). Estos resultados confirman la presencia de los orbivirus en animales de vida silvestre en América Latina.

El EHDV afecta a rumiantes domésticos y a varias especies de venados en los que produce una severa enfermedad, similar a lengua azul (MacLachlan y Guthrie, 2010); sin embargo, no existe evidencia serológica ni clínica previa de la presencia de EHD en el país, pero las diferencias encontradas con el uso de estos kits parecieran indicar que está presente en el trópico peruano. Tanto el EHDV como el VLA están presentes en muchos países del mundo y coincide con la distribución de los insectos vectores del género Culicoides competentes y con las condiciones climáticas apropiadas (Tabachnick, 2010; MacLachlan, 2011; Savini et al., 2011). El departamento de Madre de Dios, lugar de procedencia de las huanganas, posee un ecosistema que es favorable para la proliferación de estos insectos vectores. Asimismo, es posible que la infección de las huanganas por ambos agentes virales haya sido de tipo subclínica, ya que los animales estuvieron aparentemente normales en el momento del muestreo. 
Cuadro 1. Frecuencia de anticuerpos contra el virus Lengua Azul (VLA) y contra otros orbivirus en huanganas (Tayassu pecari) de vida libre en tres localidades de Madre de Dios, detectadas mediante la prueba ELISA de competición e Inmunodifusión

\begin{tabular}{lccccc}
\hline \multirow{2}{*}{ Procedencia } & $\begin{array}{c}\text { Animales } \\
(\mathrm{n})\end{array}$ & \multicolumn{2}{c}{$\begin{array}{c}\text { Animales con anticuerpos } \\
\text { específicos contra VLA }\end{array}$} & \multicolumn{2}{c}{$\begin{array}{c}\text { Animales con anticuerpos } \\
\text { contra VLA /otro Orbivirus }\end{array}$} \\
\cline { 3 - 6 } & & $\mathrm{n}$ & $\%$ & $\mathrm{n}$ & $\%$ \\
\hline Boca de Manu & 44 & 2 & 4.5 & 10 & 22.7 \\
Los Amigos & 14 & 1 & 7.1 & 4 & 28.6 \\
Tambopata & 48 & 5 & 10.4 & 17 & 35.4 \\
\hline Total & 106 & 8 & 7.5 & 31 & 29.2 \\
\hline
\end{tabular}

Se conocen 26 serotipos con distintos grados de virulencia del VLA (Madani et al., 2011; Coetzee et al., 2012) y la distribución de cada serotipo depende de los ecosistemas donde estos serotipos han evolucionado con sus respectivos vectores (Gibbs y Greiner, 1994). Las regiones del Caribe y América Central presentan los ecosistemas ideales para la convivencia de los serotipos del VLA y sus respectivos vectores Culicoides (Martínez y Becerra, 2011). Más de 1400 especies del género Culicoides han sido reportados en el mundo pero solo cerca de 30 de ellos han sido implicados en la transmisión del VLA y del EHDV (Tabachnick, 2010; Coetzee et al., 2012).

La EHDV está igualmente distribuida en todo el mundo incluyendo las Américas, África, Australia y Asia (MacLachlan y Guthrie, 2010). Este serogrupo posee ocho serotipos y algunos de ellos causan serias enfermedades en animales domésticos y silvestres. Ambos virus, VLA y EHDV, inducen anticuerpos precipitantes detectados mediante la prueba de inmunodifusión, de allí que la diferenciación se tenga que hacer mediante la prueba de ELISA de competición o mediante técnicas moleculares (Kedmi et al., 2011; Madani et al., 2011). La distri- bución de ambos virus son usualmente estudiados en forma simultánea por lo que los datos de distribución del VLA puede servir para determinar el riesgo de exposición al EHDV y viceversa. Es así que a mayor prevalencia del VLA, existen más probabilidades de encontrar animales seropositivos al EHDV (Kedmi et al., 2011; Savini et al., 2011).

La Organización Mundial para la Salud (OMS) y muchos investigadores estiman que el cambio climático global tendrá un enorme impacto en la salud del hombre y de los animales debido a la emergencia o reemergencia de enfermedades transmitidas por insectos vectores (Tabachnick, 2010). Un ejemplo es la ocurrencia de severos brotes de lengua azul en varios países europeos debido a las incursiones de los vectores competentes en latitudes donde antes no existían (Panagiotatos, 2004; García-Bocanegra et al., 2011). Ante esta perspectiva y desde el punto de vista de las ciencias veterinarias, es necesario conocer mejor la epidemiología de la lengua azul, identificar los serotipos existentes en el país, así como las especies del género Culicoides transmisores del VLA que han sido descritos (Lager et al., 2004). Desde que los signos clínicos del VLA son indistinguibles del 
EHDV en rumiantes silvestres y domésticos, la OIE (2008) recomienda incluir en el diagnóstico diferencial al EHDV mediante técnicas de laboratorio específicos.

\section{Agradecimientos}

Los autores agradecen a la Dirección General Forestal y de Fauna Silvestre del Ministerio de Agricultura y a la Jefatura de la Reserva Nacional de Tambopata y Parque Nacional Bahuaja Sonene del SERNANP por facilitar los permisos para la colecta de muestras en animales de vida libre. Así mismo, al Dr. Miguel Ara por sus comentarios sobre el manuscrito.

\section{Literatura Citada}

1. Chandel BS, Chauhan HC, Kher HN. 2003. Comparison of the standard AGID test and competitive ELISA for detecting bluetongue virus antibodies in camels in Gujarat, India. Trop Anim Health Prod 35: 99-104.

2. Clavijo A, Sun F, Lester T, Japerson TL, Wilson WC. 2010. An improved real time reverse transcriptase polymerase chain reaction for the simultaneous detection of all serotypes of epizootic hemorrhagic disease virus. J Vet Diagn Invest 22: 588-593.

3. Coetzee P, Van Vuuren M, Stokstad M, Myrmel M, Venter EH. 2012. Bluetongue virus genetic and phenotypic diversity: towards identifying the molecular determinats that influence virulence and transmission potential. Vet Microbiol 161: 1-12.

4. Congreso de la República, Comisión de Comercio Exterior y Turismo. 2004. El Perú: Un país megadiverso. I Taller de Competitividad del Sector Ecoturismo en Madre de Dios. [Internet], [27 diciembre 2011]. Disponible en: http://www. sernanp. gob.pe/sernanp/archivos/imagenes/vida/ Marco_Teorico\% 20congreso.pdf
5. Della-Porta AJ, Parsonson IM, Mcphee AD. 1985. Problems in the interpretation of diagnostic test due to cross-reaction between Orbivirus and broad serological responses in animals. Prog Clin Biol Res 178: 445-453.

6. García-Bocanegra I, Arenas-Montes A, Lorca-Oró C, Pujols J, González MA, Napp S, Gómez-Guillamón F, et al. 2011. Role of wild ruminants in the epidemiology of bluetongue virus serotypes 1, 4 and 8 in Spain. Vet Res 42: 88-94.

7. García I, Napp S, Casal J, Perea A, Allepuz A, Alba A, Carbonero A, et al. 2009. Bluetongue epidemiology in wild ruminants from Southern Spain. Eur J Wildl Res 55: 173-178.

8. Gerber PF, Galinari GCF, Cortez A, Paula CD, Lobato ZIP, Heinemann MB. 2012. Orbivirus infections in collared peccaries (Tayassu tajacu) in Southeastern Brazil. J Wildl Dis 48: 230232.

9. Gibbs EP, Greiner EC. 1994. The epidemiology of bluetongue. Comp Immunol Microbiol Infect Dis 17: 207220.

10. Gorman BM. 1986. Evolutionary relationships among orbivirus. Rev Sci Tech Off Int Epiz 5: 323-332.

11. Herrera H, Abreu U, Keuroghlian A, Freitas T, Jansen AM. 2008. The role played by sympatric collared peccary (Tayassu tajacu), white-lipped peccary (Tayassu pecari), and feral pig (Sus scrofa) as maintenance hosts for Trypanosoma evansi and Trypanosoma cruzi in a sylvatic area of Brazil. Parasitol Res 103: 619-624.

12. Kedmi M, Galon N, Herziger Y, Yadin H, Bombarov V, Batten C, et al. 2011. Comparison of the epidemiology of epizootic haemorrhagic disease and bluetongue viruses in dairy cattle in Israel. Vet J 190: 77-83.

13. Lager IA. 2004. Bluetongue virus in South América: overview of virus, vectors, surveillance and unique features. Vet Ital 40: 89-93. 
14. Lager IA, Duffy S, Miquet J, Vagnozzi A, Gorchs C, Draghi M, Cetrá B, et al. 2004. Incidence and isolation of bluetongue virus infection in cattle of the Santo Tomé Department, Corrientes Province, Argentina. Vet Ital 40: 141-144.

15. MacLachlan NJ. 2004. Bluetongue: pathogenesis and duration of viraemia. Vet Ital 40: 462-467.

16. MacLachlan NJ. 2011. Bluetongue: history, global epidemiology, and pathogenesis. Prev Vet Med 102: 107111.

17. MacLachlan NJ, Guthrie AJ. 2010. Re-emergency of bluetongue, African horse sickness, and other Orbivirus diseases. Vet Res 41(6): 35. doi: 10. 1051/ vetres / 2010007

18. Madani H, Casal J, Alba A, Allepuz A, Cetre-Sossah C, Hafsi L, KountChareb H, et al. 2011. Animal diseases caused by orbiviruses, Algeria. Emerg Infect Dis 17: 2325-2327.

19. Martínez N, Becerra AM. 2011. Primer reporte de bovinos seroreactores al virus lengua azul en Cuba. Rev Salud Anim 33: 131-133.

20. Mertens PPC, Diprose J, Maan S, Singh KP, Attoui H, Samuel AR. 2004. Bluetongue virus replication, molecular and structural biology. Vet Ital 40: 426437.

21. [OIE] Organización Mundial de Sanidad Animal. 2008. Manual de las pruebas de diagnóstico y de las vacunas para los animales terrestres. Parte 2, Sección 2.1, Capítulo 2.1.9. Francia: OIE.

22. Panagiotatos DE. 2004. Regional overview of bluetongue viruses, vectors, survillance and unique features in Europe between 1998 and 2003. Vet Ital 40: 61-72.

23. Painter L. 1996. Aspectos de salud poblacional y la biología reproductiva del pecarí de labio blanco, Tayassu pecari, en el Parque Nacional Noel Kempff Mercado. Bol Bolfor 9: 11-14.

24. Pearson JE, Carbrey EA, Gustafson GA. 1985. Bluetongue and related orbivirus diagnosis in the United States. Prog Clin Biol Res 178: 469-475.
25. Ristow S, Leendersten L, Gorham J, Yilma T. 1988. Identification of a neutralizing epitope shared by bluetongue virus serotypes 2 and 13. J Virol 62: 2502-2504.

26. Rivera H, Madewell BR, Ameghino E. 1987. Serological survey of viral antibodies in the Peruvian alpaca (Lama vicugna). Am J Vet Res 48: 189-191.

27. Romero M. 2010. Evaluación sanitaria de la presencia de enfermedades y caracterización de los patrones de caza de subsistencia de la huangana (Tayassu pecari) de vida libre en la Amazonía peruana. Tesis de Maestría. Sao Paulo, Brasil: Universidade de Sao Paulo. 62 p.

28. Romero M, Lí O, Gálvez J, Elías $R$, Alvarado A. 2008. Niveles referenciales séricos de urea y creatinina em huanganas (Tayassu pecari) criados en un zoológico de Lima. Rev Inv Vet Peru 19: 79-81.

29. Rosadio RH, Evermann JF, DeMartini JC. 1984. A preliminary serological survey of viral antibodies in Peruvian sheep. Vet Microbiol 10: 9196.

30. Savini G, Afonso A, Mellor P, Aradaib I, Yadin H, Sanaa M, Wilson W, et al. 2011. Epizootic haemorragic disease. Res Vet Sci 91: 1-17.

31. Stallknecht DE, Luttrell MP, Smith KE, Nettles VF. 1996. Hemorrhagic disease in white tailed deer in Texas: a case for endemic stability. J Wildlife Dis 32: 695700 .

32. Tabachnick WJ. 2010. Challenges in predicting climate and environmental effects on vector-borne disease episystems in a changing world. J Exp Biol 213: 946-954.

33. Tabachnick WJ. 2004. Culicoides and the global epidemiology of bluetongue virus. Vet Ital 40: 145-150.

34. Tobler MW, Carrillo-Percastegui SE, Powell G. 2009. Habitat use, activity patterns and use of mineral licks by five species of ungulate in south eastern of Peru. J Trop Ecol 25: 261-270. 